\title{
Creep Behaviour of Co-Based Amorphous Alloys
}

\author{
K. Csach, J. MišKuf, A. Juríková \\ Institute of Experimental Physics, Slovak Academy of Sciences \\ Watsonova 47, 04001 Košice, Slovakia \\ AND P. VOJTANÍK \\ Institute of Physics, Faculty of Science, P.J. Šafárik University \\ Park Angelinum 9, 04001 Košice, Slovakia
}

The detailed study of the temperature dependence of the viscosity of $(\mathrm{Co}, \mathrm{Fe})-(\mathrm{Si}, \mathrm{B})$ amorphous metallic alloys up to crystallization temperatures was carried out. The kinetics of the non-equilibrium viscosity was described using Vogel-Fulcher-Tammann law below the glass transition temperature.

PACS numbers: 61.43.Dq, 62.20.Hg

\section{Introduction}

Amorphous metallic glasses based on transition metals are in the center of the interest due to their useful mainly magnetic properties. Especially Co-based alloys are interesting for their magnetoelastic behaviour $[1,2]$. The plastic deformation ability is very important property for the practical exploitation of these materials. The magnetic properties are sensitive to mechanical stresses and structural defects. Stress sensitive defects play the important role in the forming ability of these alloys. The homogeneous (i.e. creep) deformation is controlled by the creation and anihilation of defects and their mobility. For liquids as well as for undercooled liquids the model for the temperature dependence of a viscosity $\eta(T)$ in equilibrium condition is usually used $[3,4]$. The empirical Vogel-Fulcher-Tammann (VFT) equation is the Arrhenius law modified by terms of some constants $B$ and $T_{0}$ that reflect the temperature dependence of a deformation defect concentration

$$
\eta(T)=\eta_{0} \exp \frac{B}{T-T_{0}} .
$$

Amorphous metals below the glass transition temperature are in a metastable state and the non-equilibrium viscosity is observed $[4,5]$. Structural relaxation effects influence the structure of amorphous alloys towards more stable state [6]. The 
aim of this work is to examine the temperature dependence of the non-equilibrium viscosity of some metal-metalloid glasses and to check the validity of VFT formula for these alloys at temperatures below the glass transition.

\section{Experimental details}

Amorphous metallic ribbons $\mathrm{Co}_{82} \mathrm{~B}_{18}, \mathrm{Co}_{75} \mathrm{Si}_{15} \mathrm{~B}_{10}, \mathrm{Co}_{37.5} \mathrm{Fe}_{37.5} \mathrm{Si}_{15} \mathrm{~B}_{10}$ and $\mathrm{Fe}_{75} \mathrm{Si}_{15} \mathrm{~B}_{10}$ in as-quenched state were used in experiments. Uniaxial tensions of $15 \mathrm{MPa}$ and $150 \mathrm{MPa}$ were applied during the linear heating with the rate of $10 \mathrm{~K} / \mathrm{min}$ and the elongation of samples was measured using the thermomechanical analyser Setaram TMA92 with the resolution of $0.01 \mu \mathrm{m}$. A flow of pure argon atmosphere was used to protect samples. The viscosity was determined using the method described in [7]. This method is based on measuring of the sample elongation $\Delta l(t, T, \sigma)$ during the linear heating under different stresses $\sigma$ :

$$
\Delta l(t, T, \sigma)=l_{0} \int_{T_{0}}^{T} \alpha(T) \mathrm{d} T+l_{0} \sigma \int_{T_{0}}^{T} \frac{\alpha(T)}{E(T)} \mathrm{d} T+l(T) K \sigma \int_{0}^{t(T)} \frac{1}{\eta(T)} \mathrm{d} t .
$$

The first term corresponds to the thermal dilatation of the sample with the length $l_{0}$ and the coefficient of thermal expansion $\alpha(T)$. The second term is given by the elasticity under an applied stress $\sigma$. The last term reflects the flow of the sample under an applied stress, $K$ is a numerical constant. Considering the small temperature dependence of elastic properties it is possible to eliminate the first and the second term in Eq. (2) by repeating the identical experiment in tension under low and high stresses. The creep deformation $\varepsilon$ was obtained by the subtraction of length changes $\Delta l$ obtained under the high stress from the ones obtained under the low stress. Finally, the shear viscosity was calculated using the deformation rate $\dot{\varepsilon}$ as $\eta=\Delta \sigma / 3 \dot{\varepsilon}$.

\section{Results and discussion}

The thermal elongation of samples measured under the low applied stress is shown in Fig. 1. During the linear heating the structural relaxation occurs and a deviation from the simple thermal dilatation is visible. For all samples besides CoB the crystallization is observed as a contraction. Only the temperature interval up to the crystallization temperature onset was taken into account for further analysis.

The measured temperature dependences of $\eta$ as well as the corresponding fits according to VFT equation can be seen in Fig. 2. It is shown that the viscosity of $\mathrm{CoB}$ alloy in the whole examined temperature interval is higher than for other samples. Increasing the content of both metalloids ( $\mathrm{Si}$ and B) from $18 \%$ to $25 \%$ leads to the decrease in the viscosity $\eta$ due to the increased amount of more mobile defects. Higher stability of $\mathrm{CoB}$ alloy is argued by the fact that during the heating up to the temperature of $830 \mathrm{~K}$, the amorphous structure is saved whereas the amorphous alloys with other compositions undergo the crystallization. 


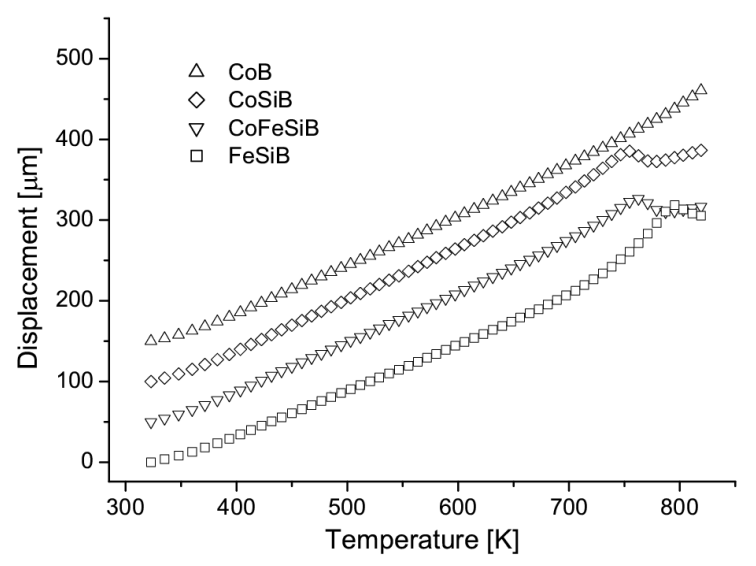

Fig. 1. Dilatation curves measured under the low stress of $15 \mathrm{MPa}$.

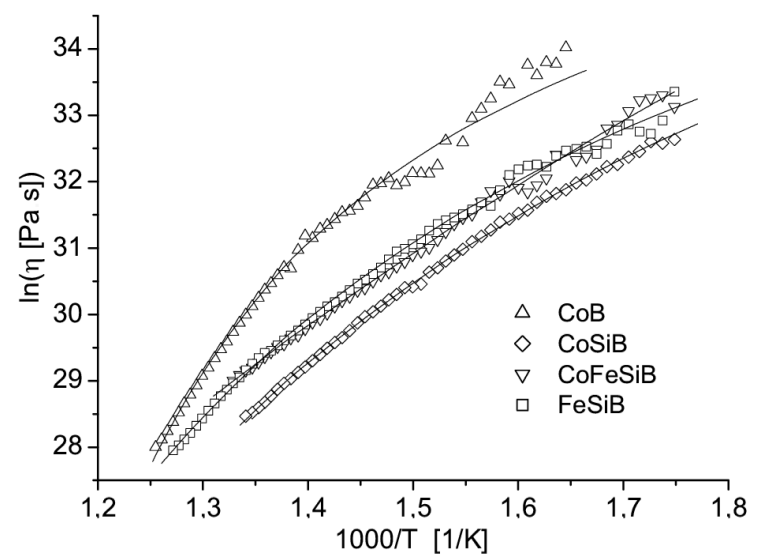

Fig. 2. The measured viscosity $\eta$ (symbols) and VFT fits (lines).

For amorphous alloys FeSiB the lowest viscosity is observed. The partial substitution of Fe atoms by Co increases the viscosity $\eta$ at all temperatures. Additional substitution of all atoms Fe by Co does not influence the creep behaviour significantly. The viscosity of CoSiB alloy is lower than for CoB alloy. This phenomenon can be caused by the different content of metalloids and their nature. It should be noted that the direct conclusion about the influence of the metalmetalloid type bonds on the creep behaviour cannot be made due to the fact that the structure of the used as-quenched samples is metastable up to the temperatures below the glass transition temperature.

\section{Summary}

The detailed study of the temperature dependence of the viscosity of $(\mathrm{Co}, \mathrm{Fe})-(\mathrm{Si}, \mathrm{B})$ amorphous alloys at temperatures up to crystallization temperatures has been carried out. It was shown that the non-equilibrium viscosity can 
be described by the Vogel-Fulcher-Tammann law. The increase in the metalloid content in amorphous alloys causes the decrease in their viscosity. The FeSiB alloy has lower viscosity than $\mathrm{CoSiB}$ with the identical content of metalloids.

\section{Acknowledgments}

The work was supported by the Slovak Grant Agency for Science - VEGA.

\section{References}

[1] D.C. Jiles, Acta Mater. 51, 5907 (2003).

[2] P. Vojtaník, J. Magn. Magn. Mater. 304, 159 (2006).

[3] I. Avramov, J. Non-Cryst. Solids 351, 3163 (2005).

[4] K. Russew, Mater. Sci. Eng. A 226-228, 779 (1997).

[5] V.A. Khonik, J. Non-Cryst. Solids 296, 147 (2001).

[6] K. Csach, V. Ocelík, IEEE Trans. Magn. 30, 496 (1994).

[7] G. Vlasák, P. Švec, P. Duhaj, Mater. Sci. Eng. A 304-306, 472 (2001). 